\title{
An Embedded Secondary Contention Window for In-Band Full-Duplex Enabled WLAN
}

\author{
Wen-Nai Wang, Xin-Meng Wang \\ Key Lab of Broadband Wireless Communication and Sensor \\ Network Technology, Ministry of Education \\ Nanjing University of Posts and Telecommunications \\ Nanjing, China \\ E-mail:wangwn@njupt.edu.cn, $1530410941 @ q q . c o m$
}

\author{
Wei $\mathrm{Wu}$ \\ School of Communications and Information Engineering \\ Nanjing University of Posts and Telecommunications \\ Nanjing, China \\ E-mail: wdepingfang@ foxmail.com
}

\begin{abstract}
In-band full-duplex wireless techniques have provided an attractive opportunity to double both spectrum efficiency and network throughput. A shared wireless channel permits two sets of directional paths, one called by primary and the other by secondary, onto which two different pair of wireless stations can send simultaneously their packets with no collision. The complexity emerges in station selection for multiple access due to random distribution, especially for WLAN. We propose in this paper an elaborate scheme to address the competition in secondary path access while keeping primary path fully compatible to the conventional 4-way handshaking of IEEE 802.11 Distributed Coordination Function (DCF). A generic framework of throughput analysis is discussed and a formulation specific to a simplified topology is deduced. Numeric evaluations show that the proposed scheme promises a maximum speedup factor beyond double in magnitude.
\end{abstract}

Keywords-in-band full-duplex; WLAN; secondary path access; embedded contention window; p-consistence CSMA; throughput performance

\section{INTRODUCTION}

Recent advances in wireless in-band Full-Duplex (FD) techniques have provided an attractive opportunity to double not only spectrum efficiency but also transmission throughput in comparison with half-duplex (HD) counterpart [1]. The common and shared wireless channel permits two packets being transmitted simultaneously by two neighbor stations in case each is capable of self-interference cancellation. However, this good feature cannot be utilized directly for a multiple random access network such as conventional WLAN. The complexity of contention collision on channel and also hidden node effect should be re-examined and re-devised elaborately and properly.

An FD channel shared by two wireless stations can employ two transmission paths with opposite direction [2]. This simplest topology is referred to as bi-directional pair. As for WLAN applications, a relay-like topology emerges where two mutual hidden stations are reachable to the third one, and can form two co-directional accessible paths [3], or a chain-typed structure, as depicted in Fig.1.

In Fig.1, we assume all stations are FD enabled and their receiving ( $\mathrm{Rx}$ ) and transmitting (Tx) blocks plotted in a split manner and filled by different color. The station A is assumed in further to transmit a data frame to it neighbor $\mathrm{B}$, and the station $\mathrm{C}$ and $\mathrm{D}$ are hidden to $\mathrm{A}$ and $\mathrm{B}$, respectively, due to distance fading or obstacle screening.

From Fig.1, one can find three types of accessible path combination. Using the note (A, B) for the primary path, the secondary path can be denoted by $(\mathrm{B}, \mathrm{A})$ for bi-directional pair, $(\mathrm{B}, \mathrm{C})$ for forward chain, and (D, A) for backward chain. However, the three secondary paths are mutual exclusion, i.e., only one of them can be selected in order to avoid the unresolvable interference or collision in frames transmission. Meanwhile, any exposed station like the node E in Fig.1 must be excluded in the selection for that such station will disturb inevitably the primary path transmission.

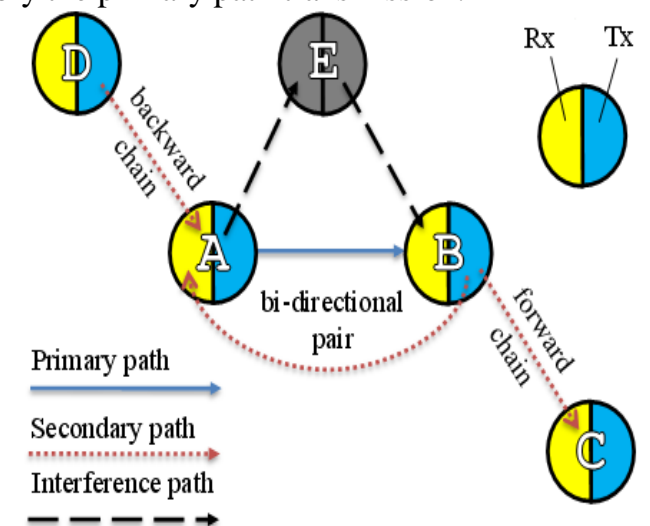

Figure 1. Illustration of 3 types of path structure of FD channel.

Generally, the selection of primary and secondary path can be carried out either simultaneously or sequentially. A simultaneous manner requires all participants support fullduplex operations [4], while a sequential one is easy to work with legacy HD stations. In this paper, we present a design of sequential scheme which consists of two stages, the first adopting 4-way handshaking DCF for primary path and the second using p-persistent CSMA for secondary path. The key to scheme is to embed a so-called Secondary Contention Window (SCW) by Network Allocation Vector (NAV) of primary RTS control frame in its duration field.

The rest of this paper is organized as follows: Section II describes our scheme design. Section III presents a generic analysis on the performance of throughput and related HD compatibility. Results of numeric evaluation are presented in Sections IV, followed by a discussion and conclusion section. 


\section{SCHEME DESIGN}

Our scheme is based on a wireless station with FD enabled physical layer, and focuses on Media Access Control (MAC) layer to select efficiently secondary path. In order to make it feasible to practical deployment, the scheme, named by fullduplex DCF (FDCF), is set to work with conventional station with $\mathrm{HD}$ operations.

Aiming at HD compatible, RTS/CTS handshaking defined in IEEE 802.11 DCF remains unchanged but a bit of modification on NAV computation as showed in Fig.2. An example of frames flow is described for a forward chain, in comparison with that of conventional DCF. The cases of bidirectional pair and backward chain are the same except an extra delay in secondary path contention in the latter.

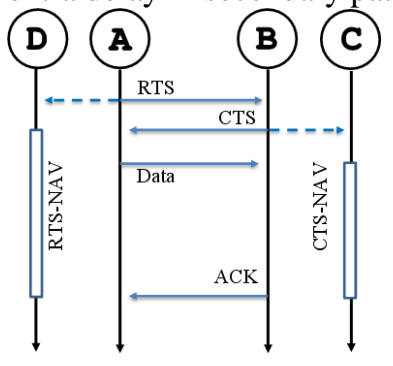

(a)

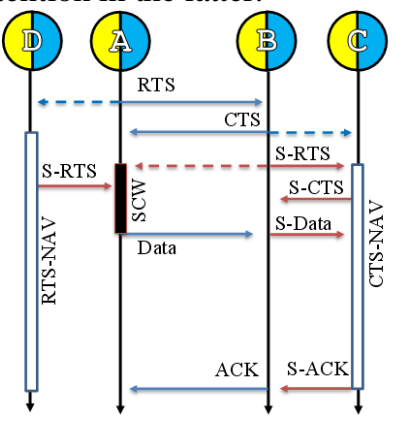

(b)
Figure 2 The frame flow of 4-way handshaking DCF (a), and FDCF (b).

In Fig.2(b), a time interval indicated by a solid bar is for SCW embedded in NAV of primary RTS. During the interval, stations contend to secondary path send a Secondary RTS (SRTS), and wait to receive Secondary CTS (S-CTS) response from its addressed receiver. The handshaking of extra S-RTS and S-CTS is introduced here to control multiple access by the method similar to DCF. The specification of conventional RTS and CTS frames remains no change for S-RTS and S-CTS, respectively.

The SCW interval covers in time both S-RTS and S-CTS transmission, plus some necessary inter-frame spaces. At the end of SCW, double data frames, as denoted by Data and SData in Fig.2, are sent simultaneously onto the primary and secondary path, respectively. On successfully received, double ACK frames are acknowledged also simultaneously.

Comparing with conventional DCF, the FDCF will take a little more time, i.e., a SCW, to complete its data frame(s) transmission. This overhead can be compensated in the performance of throughput by the doubling of data frame transmission if the secondary path can be accessed without any interference or collision.

It can be imagined that collision happens if more than one station, such as the node B, C and D in Fig.1, contends the secondary path. Also, there is a probability the receiver of the secondary path fails to response to S-RTS which is addressed to the exposed node such as E. These two cases result in a degradation in effective utilization and throughput. We propose in the following a p-consistent CSMA technique to reduce the contention collision on secondary path.

At the beginning of SCW, the primary receiver, represented by the node B in Fig.1, delays its S-RTS by a PIFS. The reason of PIFS choosing other than SIFS is for HD compatible and will be explained in the latter. Others similar to the node D delay their S-RTS by a longer time to let the node B complete its S-RTS transmission. The long delay is used to avoid any potential interference to the receiving at the node A the S-RTS from B. Therefore, the long delay of is set as PIFS plus TRTS, the time of one RTS frame transmission.

The choosing of two different delays before S-RTS sending depends solely on the sender whether it has been sensed a primary RTS addressed to it or not. As the example showed in Fig.2(b), the node B must choose a short delay, and the node D a long delay.

The primary sender A responses by a S-CTS frame only under two conditions, one for S-RTS sent from B to A, the other for S-RTS sent from D to A. In case B contends secondary path by a S-RTS addressed to the node $\mathrm{C}$ rather than the node A, the primary sender should not response any S-CTS, and $\mathrm{D}$ will exit the sequential contention procedure. In other words, forward chains have priority over backward chains in secondary path access.

For multiple nodes similar to $\mathrm{D}$, there are still multiple backward chains joining the secondary path access. We propose to adopt the conventional p-consistent CSMA method by assigning a probability of S-RTS sending. The probability for $\mathrm{D}$ can be optimized dynamically according to $\mathrm{N}_{\mathrm{D}}$, the number of backward chains. For simplicity, we set the probability, $\mathrm{p}=1 / \mathrm{N}_{\mathrm{D}}$, as defined in [5].

HD compatibility of the FDCF is straightforward. For the case the primary sender, A, operates in HD mode, it starts to send its waiting data frame at the very end of SIFS after CTS transmission. Since the delay of S-RTS has been defined as PIFS or PIFS $+T_{R T S}$, longer than SIFS, any attempt to secondary path will quit and result in no interference to A in $\mathrm{HD}$ mode. Actually, for a primary sender in HD mode, the proposed FDCF goes back to conventional DCF.

For the primary receiver, $\mathrm{B}$, operating in $\mathrm{HD}$ mode, it will block data transmitting procedure according to DCF specification until the end of RTS-NAV indicated by the primary sender A. The only difference between FDCF and DCF in this case is the unemployed time of SCW. The little low utilization result is also valid for the case the node D operating in HD mode.

\section{PERFORMANCE ANALYSIS}

The probability of successful channel contending for conventional DCF is denoted by $\mathrm{p}_{\mathrm{DCF}}$. Generally, this probability represents the condition only one pair of stations starting RTS/CTS handshaking, referring [6], as follows,

$$
P_{\mathrm{DCF}}=\frac{n q(1-q)^{n-1}}{1-(1-q)^{n}} \text {, }
$$

where, $\mathrm{n}$ is the number of stations contending on the common channel with the same probability q of RTS sending.

Given that, there is a probability, $1-\mathrm{P}_{\mathrm{DCF}}$, an RTS/CTS handshaking disturbed and interrupted by collision.

As for FDCF, a primary receiver, the node B in Fig.1 and Fig2, can always succeed in contending secondary path if it has waiting data addressed to the primary sender $\mathrm{A}$, i.e., $\mathrm{p}_{\mathrm{BA}}=1$.

Also, the succeed probability on the path from the node $\mathrm{B}$ to $C$ is direct, $\mathrm{p}_{\mathrm{BC}}=\mathrm{N}_{\mathrm{C}} /\left(\mathrm{N}_{\mathrm{C}}+\mathrm{N}_{\mathrm{E}}\right)$, assuming $\mathrm{B}$ select 
randomly its neighbor nodes other than $A$, and $N_{E}$ and $N_{C}$ denoting the number of exposed nodes and that of accessible forward chains, respectively.

By the same consideration, we denote the number of accessible backward chains by $\mathrm{N}_{\mathrm{D}}$. It is equal to the number of nodes similar to the node D in Fig.1. The succeed probability on the path from $\mathrm{D}$ to $\mathrm{A}$, given that the node $\mathrm{B}$ has no data waiting to send, is then,

$$
p_{\mathrm{DA}}=(1-p)^{N_{D}-1}=\left(1-\frac{1}{N_{D}}\right)^{N_{D}-1},
$$

where, $\mathrm{N}_{\mathrm{D}}$ is assumed greater then 0 , otherwise $\mathrm{p}_{\mathrm{DA}}=0$. Again we use the assumption $\mathrm{p}=1 / \mathrm{N}_{\mathrm{D}}$, as mentioned before.

The total probability of the collision-free secondary path contending is then the sum as follows,

$$
\begin{aligned}
P_{\mathrm{SP}}= & q_{\mathrm{BA}} p_{\mathrm{BA}}+\left(1-q_{\mathrm{BA}}\right) q_{\mathrm{BC}} p_{\mathrm{BC}} \\
& +\left(1-q_{\mathrm{BA}}\right)\left(1-q_{\mathrm{BC}}\right) q_{\mathrm{DA}} p_{\mathrm{DA}},
\end{aligned}
$$

where, $\mathrm{q}_{\mathrm{BA}}$ is the probability of the node $\mathrm{B}$ has data waiting to send to $A, q_{B C}$ is that from $B$ to $C$, and $q_{D A}$ that from $D$ to $A$.

As one can see, $\mathrm{q}_{\mathrm{BA}}$ is equal to the average request, $\mathrm{q}$, defined in Eq.(1), between any pair of nearest neighbors. Under a fully randomized traffics, one can derive the following two equations,

$$
\begin{gathered}
q_{\mathrm{BC}}=1-(1-q)^{N_{\mathrm{C}}+N_{\mathrm{E}}}, \\
q_{\mathrm{DA}}=1-(1-q)^{N_{\mathrm{D}}} .
\end{gathered}
$$

For a simple case, let $\mathrm{N}_{\mathrm{C}}=1, \mathrm{~N}_{\mathrm{E}}=0$ and $\mathrm{N}_{\mathrm{D}}=1$, a 4-node chain-like topology, it can get then,

$$
\begin{aligned}
& q_{A B}=q_{B C}=q_{D A}=q, \\
& p_{B A}=p_{B C}=p_{D A}=1 .
\end{aligned}
$$

By replacing Eq.(6) and (7) into Eq.(3), we have,

$$
P_{g P}=q+(1-q) q+(1-q)^{2} q=q\left(3-3 q+q^{2}\right)
$$

That means, there is probability $\mathrm{q}\left(3-3 \mathrm{q}+\mathrm{q}^{2}\right)$ in the 4-node chain-like topology to send two packets within one successful transmission period, $\mathrm{T}_{\mathrm{FDCF}}$, headed by RTS/CTS handshaking.
Since our FDCF scheme has only an embedded SCW interval in comparison with the conventional DCF, it can be represented by,

$$
T_{\mathrm{FDCF}}=T_{\mathrm{DCF}}+T_{\mathrm{SCW}}
$$

Assuming all packets is fixed in length, Ldata, and requires the time Tdata to transmit them, it is then,

$$
\mathrm{T}_{\mathrm{DCF}}=\mathrm{T}_{\mathrm{RTS}}+\mathrm{T}_{\mathrm{CTS}}+\mathrm{T}_{\mathrm{data}}+\mathrm{T}_{\mathrm{ACK}}+\mathrm{DIFS}+3 \times \mathrm{SIFS}
$$

where, $\mathrm{T}_{\mathrm{RTS}}, \mathrm{T}_{\mathrm{CTS}}$ and $\mathrm{T}_{\mathrm{ACK}}$ are, in turn, the time to transmit the frame of RTS, CTS and ACK, DIFS for the time for channel idle sensing, and SIFS for inter-frame spacing among the four frames.

The time, $\mathrm{T}_{\mathrm{SCW}}$, is determined by the longest reserved interval for the node D in Fig.1, including the time to transmit two RTSs, one CTS, the leading PIFS and two SIFSs, that is,

$$
\mathrm{T}_{\mathrm{SCW}}=2 \times \mathrm{T}_{\mathrm{RTS}}+\mathrm{T}_{\mathrm{CTS}}+\mathrm{PIFS}+2 \times \mathrm{SIFS}
$$

The average time spent for unsuccessful contention in DCF can be expressed as, approximately,

$$
\mathrm{T}_{\mathrm{OH}}=\mathrm{T}_{\mathrm{RTS}}+\mathrm{T}_{\mathrm{CTS}}+\mathrm{DIFS}+\mathrm{SIFS}
$$

The average throughput of DCF is approximately,

$$
S_{D C F}=\frac{P_{\mathrm{DCF}} \times L_{\mathrm{data}}}{T_{\mathrm{OH}}\left(1-p_{\mathrm{DCF}}\right)+T_{\mathrm{DCF}} \times p_{\mathrm{DCF}}},
$$

where the average back-off time to channel access is regarded as so small to be considered, The counterpart of FDCF is then,

$$
S_{F D C F}=\frac{\left(1+P_{\mathrm{SP}}\right) \times P_{\mathrm{DCF}} \times L_{\mathrm{data}}}{T_{\mathrm{OH}}\left(1-p_{\mathrm{DCF}}\right)+T_{\mathrm{FDCF}} \times p_{\mathrm{DCF}}}
$$

The ratio of the throughput of FDCF over that of DCF, or speedup factor, is,

$$
f=\frac{S_{\mathrm{FDCF}}}{S_{\mathrm{DCF}}}=\left(1+P_{\mathrm{SP}}\right) \frac{T_{\mathrm{OH}}\left(1-P_{\mathrm{DCF}}\right)+T_{\mathrm{FDCF}} \times P_{\mathrm{DCF}}}{T_{\mathrm{OH}}\left(1-P_{\mathrm{DCF}}\right)+T_{\mathrm{DCF}} \times P_{\mathrm{DCF}}}
$$

Eq.(14) has a closed form since both $\mathrm{P}_{\mathrm{SP}}$ and $\mathrm{P}_{\mathrm{DCF}}$ are the function of the probability $\mathrm{q}$, the average rate of data sending from one station to another. Thus, the benefit of FD operation to the throughput of WLAN can be figured out as the average loaded traffics.

\section{NUMERIC EVALUATION}

We use parameters of DCF specified in IEEE 802.11b [7] and those of FDCF are showed in Tab.I for the calculation according to Eq.(15). The length of data frame and the probability station sends the frame, are the only that affects the speedup factor. 
TABLE I. PARAMETERS USED FOR NUMERIC EVALUATIONS

\begin{tabular}{|c|c|c|c|}
\hline Parameter & Value & Parameter & Value \\
\hline Bandwidth & $11 \mathrm{Mbit} / \mathrm{s}$ & $T_{\mathrm{CTS}}$ & $10.2 \mu \mathrm{s}$ \\
\hline SIFS & $10 \mu \mathrm{s}$ & $T_{\mathrm{ACK}}$ & $10.2 \mu \mathrm{s}$ \\
\hline PIFS & $30 \mu \mathrm{s}$ & $T_{\mathrm{DCF}}$ & $114.9 \mu \mathrm{s}+T_{\mathrm{data}}$ \\
\hline DIFS & $50 \mu \mathrm{s}$ & $T_{\mathrm{SCW}}$ & $89.2 \mu \mathrm{s}$ \\
\hline RTS & $20 \mathrm{Bytes}$ & $T_{\mathrm{FDCF}}$ & $204.1 \mu \mathrm{s}+T_{\mathrm{data}}$ \\
\hline CTS/ACK & $14 \mathrm{Bytes}$ & $T_{\mathrm{data}}(64 \mathrm{~B})$ & $46.5 \mu \mathrm{s}$ \\
\hline$T_{\mathrm{RTS}}$ & $14.5 \mu \mathrm{s}$ & $T_{\mathrm{data}}(1500 \mathrm{~B})$ & $1090.9 \mu \mathrm{s}$ \\
\hline Fig.3 & & & \\
\hline
\end{tabular}

Fig.3 shows the result of speedup factor, f, varying along with the probability of data sending, q, of a four-node chaintype WLAN. The number of station, $n=4$ for PDCF by Eq.(1), and PSP is determined by Eq.(8).

From Fig.3, it can be seen that the speedup factor for both short packet (64B) and long (1500B) packet have values greater than two. And, most significantly, the maximum speedup factor of short packet is about 2.33 at $\mathrm{q}=0.4$, and that of long packet is about 2.05 at $\mathrm{q}=0.68$.

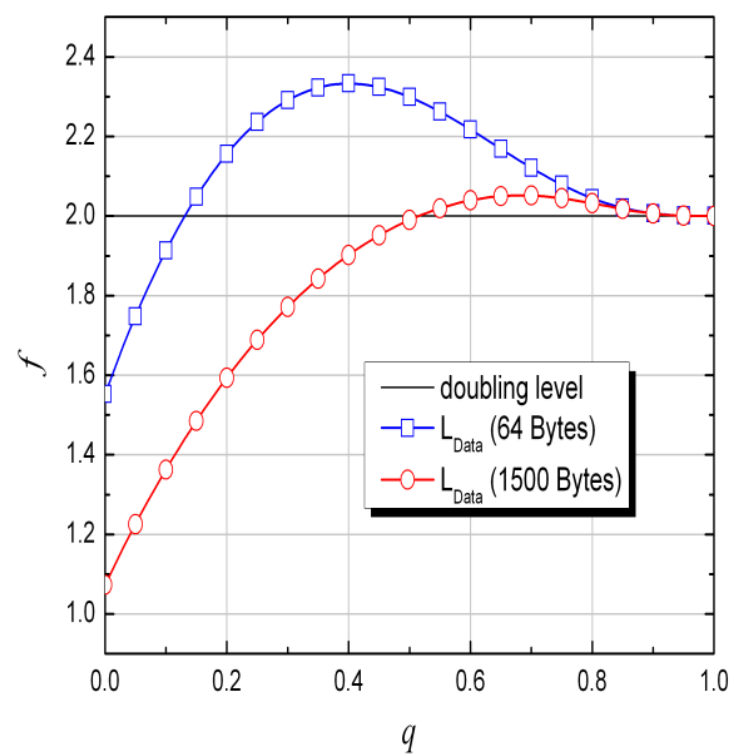

Figure 3 The throughput speedup factor $\mathrm{f}$ varies with the neighbor packet sending probability q, for an FD WLAN containing three accessible secondary paths.

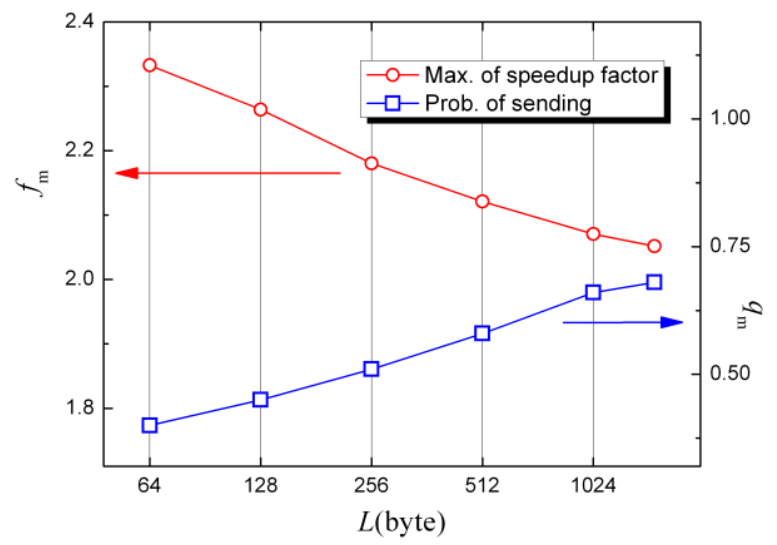

Figure 4. The maximum speedup factor fm and the correspoding sending probability qm vary with the length of packet.

Fig.4 shows a curve of maximum speedup factor varying with the length of packet, and a curve of sending probability by which the maximum factor obtains.

The reason for the throughput upgradation being greater than double relates to the reduction by FDCF in random contention.

\section{DISCUSSION AND CONCLUSION}

In-band full-duplex wireless techniques have provided an attractive approach to double both spectrum efficiency and channel capacity without needing extra radio resources. The good feature can not be utilized directly for WLAN which typically accessed randomly by multiple stations. There are three types of secondary path on a common channel leads to a more complex issue in contention collision. In this paper, we proposed a new DCF compatible scheme which focus on secondary path control of FD channel.

Specifically, a p-consistent CSMA method has been introduced in this paper to be embedded in DCF operations. The details of the proposed two stage control scheme is present followed by a closed form of performance analysis and numeric evaluations. Results show clearly a throughput speedup factor beyond double can be obtained for a simplified 4-node chain-typed topology.

The benefit of FD to upgrading WLAN throughput, we think, should be much more significant for complex topologies where much more part of contending collisions can be reduced due to secondary path employed.

\section{ACKNOWLEDGMENT}

This work has been partly supported by China National Science Foundation with grand No. 61401234.

\section{REFERENCES}

[1] D. Kim, H. Lee and D. Hong, "A Survey of In-Band Full-Duplex Transmission: From the Perspective of PHY and MAC Layers," IEEE Commun. Surveys \& Tutorials, vol. 17, no. 4, pp. 2017-2046, 2015.

[2] D. Bharadia, E. McMilin, and S. Katti, "Full duplex radios," ACM SIGCOMM Comp. Commun. Rev., vol. 43, no. 4, pp. 375-386, 2013.

[3] G. Liu, F. R. Yu, H. Ji, V. C. M. Leung and X. Li, "In-Band Full-Duplex Relaying: A Survey, Research Issues and Challenges," IEEE Commun. Surveys \& Tutorials, vol. 17, no. 2, pp. 500-524, 2015. 
[4] Wenjie Zhou, K. Srinivasan and P. Sinha, "RCTC: Rapid concurrent transmission coordination in full DuplexWireless networks," 2013 21st IEEE Intl. Conf. on Network Protocols (ICNP), pp. 1-10, 2013.

[5] R. Bruno, M. Conti, E. Gregori, "Optimal capacity of p-persistent CSMA protocols," IEEE Commun. Lett., vol. 7, pp.139-141, 2003.
[6] G. Bianchi, "Performance analysis of the IEEE 802.11 distributed coordination function," IEEE J. Sel. Areas in Commun., vol. 18, no. 3, pp. 535-547, 2000.

[7] IEEE, "Wireless LAN Medium Access Control(MAC) and Physical Layer (PHY) specifications,” IEEE Std. 802.11, 1999 Edition (R2003). 